This is an author-created, accepted version of the paper "Reducing the circadian input from selfluminous devices using hardware filters and software applications" by J. Escofet and S. Bará. The final, definitive version of this paper can be found in Lighting Research and Technology 1477153515621946, first published on December 10, 2015 as doi: 10.1177/1477153515621946, by SAGE Publications Ltd, All rights reserved. (C) The Authors

\title{
Reducing the circadian input from self-luminous devices using hardware filters and software applications
}

\author{
J Escofet $\mathrm{PhD}^{\mathrm{a}}$ and $\mathbf{S}$ Bará $\mathrm{PhD}{ }^{\mathrm{b}}$ \\ ${ }^{a}$ Departament d'Òptica i Optometria, Universitat Politècnica de Catalunya, Terrassa, \\ Catalunya, Spain

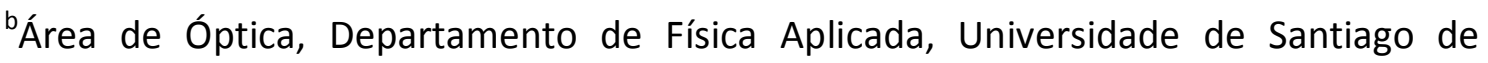 \\ Compostela, Santiago de Compostela, Galicia, Spain
}

Short title: Reducing circadian input

Received 9 September 2015; Revised 26 October 2015; Accepted 19 November 2015

The widespread use of self-luminous devices at nighttime (cell-phones, computers, and tablets) raises some reasonable concerns regarding their effects on human physiology. Light-at-night is a known circadian disruptor, particularly at short visible wavelengths, and it seems advisable to have practical tools for tailoring the spectral radiance of these displays. We analyze two possible strategies to achieve this goal, using hardware filters or software applications. Overall, software applications seem to offer, at present time, the best trade-offs for controlling the light spectra emitted by existing devices. We submit that such tools should be included as a standard feature on any selfluminous device and that their default settings should be established according to the best available knowledge on the circadian effects of light.

Address for correspondence: Salvador Bará, Área de Óptica, Dept. Física Aplicada, Facultade de Óptica e Optometría, Universidade de Santiago de Compostela, 15782 Santiago de Compostela, Galicia, Spain.

E-mail: salva.bara@usc.es 


\section{Introduction}

Portable electronic devices with self-luminous displays are increasingly present in our lives. Beyond their leisure applications, they provide effective and practical tools for social and professional networking, voice and video communication, document editing and sharing, and many other activities that were traditionally carried out using specific mid-sized desktop equipment. Smartphones, tablets and small personal computers are part of our everyday environment and significantly contribute to shape our lighting landscape, especially indoors and at nightime. It can be foreseen that their use will increase in the near future, given the fast pace of technological development in the fields of photonics and microelectronics, the existence of a growing world market for this kind of consumer electronics and the initiation into their use at ever younger ages.

The spectral composition of the light emitted by these devices has some remarkable differences with the one emitted by classical thermal or gas-discharge sources. The self-luminous screens used in many consumer electronic products are made up of a white light-emitting diode (LED) backlight source illuminating a liquidcrystal display panel (LCD) which provides the required spectral modulation to obtain the color subpixels. Organic light-emitting diodes (OLED) ${ }^{1}$ are used as well, specially in small-size screens. In all cases the light is appreciably concentrated in three broad bands in the red, green and blue regions of the visible spectrum. The frequent and prolonged use of these devices shortly before bedtime is becoming a matter of concern. The combination of relatively high radiance levels with a strong contribution from the short-wavelength region of the visible spectrum is a typical feature of these displays.

Light at night is a significant input for the circadian regulation system, ${ }^{2,3}$ and an increasing body of research is unveiling its unintended side-effects in shifting the circadian phase and suppressing pineal melatonin production. ${ }^{4-6}$ Recent results indicate that adolescents, one of the main user segments of these devices in industrial societies, may be more sensitive to these non-visual light effects than older populations. ${ }^{7}$ Although a great deal of additional work is needed to build a comprehensive quantitative model of the non-visual interactions between light and the human circadian system, several broad recommendations are gaining consensus within the scientific community. Reducing unnecessary high levels of light at night and attenuating the short-wavelength components of the spectrum are two of them. ${ }^{8-11}$ It seems then advisable to have at hand practical tools that make it possible to modify, in a controlled way, the spectral radiance of self-luminous displays in order to reduce the unwanted circadian input received by their users at nightime.

In this paper we explore two possible ways of achieving this goal, based on the use of transmission filters and of software applications. In Section 2 we briefly outline these approaches. In Section 3 we describe the parameters we used to quantify the 
circadian inputs produced by the displays. Section 4 presents two examples based on some of the displays, filters, and applications currently available. Discussion and some additional remarks are included in Section 5. Conclusions are summarized in Section 6.

\section{Tailoring the spectral radiance}

The spectral distribution of the light emitted by self-luminous displays can be controlled in several ways. A possible one, although technologically demanding, is to modify the radiant elements themselves by developing new solid-state sources whose unwanted photobiological effects can be minimized at origin, as proposed by several authors for smartphone displays ${ }^{12,13}$ and general lighting applications. ${ }^{14}$ Existing devices, however, require short-term solutions compatible with the kind of light sources they are already equipped with. Two main approaches can be used to that end: (i) using classical transmission filters that selectively attenuate certain regions of the visible spectrum or (ii) using software applications to control the relative weight of the signal emitted by each color subpixel, attenuating in that way some of the spectral bands and, if required, reinforcing others.

Conventional transmission filters are always a feasible option for modifying the spectral radiance received from a self-luminous device, in the form of protective goggles worn by the user or filters directly fitted to the displays. Relatively inexpensive broadband polymer filters can be used for a coarse tuning of the emitted spectra, and more sophisticated operations (e.g. narrow -band transmission or rejection) can be performed by using appropriately stacked dielectric multilayers. Transmission filters, however, have some basic disadvantages: they have to be physically attached to each device (or worn by the users), and since their transmittance function cannot be modified they have to be replaced each time a different filtering effect is desired. This lack of tunability is a serious drawback for their use in screens if an adaptive filtering control is required, e.g. for periodically dimming some wavelength bands depending on the timing of the circadian phase.

Software applications that control the spectral radiance of the screens by modifying the chromatic composition of the images sent to the display can be used as effective "software filters". That way the radiances of the R, G and B subpixels can be adaptively adjusted to produce the minimum circadian effects compatible with keeping some basic standards for visual quality. Software filters are intangible, do not require the consumption of materials and may be adjusted by the user to accomodate their effective transmittance to the requirements of the task to be performed, the spectral composition of the images, and the circadian phase. As a relevant drawback, since they ultimately act on the signal driving the color subpixels, they are not expected to be capable of performing very fine narrow-band spectral tunning 
operations. However, their adaptability allows for a wide control in real time of the circadian load associated with the nocturnal use of these devices.

As suggested by an anonymous reviewer, dimming the screens without changing the shape of their spectral power distributions is an additional way of attenuating the circadian effects of self-luminous devices at nightime. Dimming is formally equivalent to using a neutral density filter, and we have also explored the possibilities it may offer.

\section{Parameters for quantifying the circadian inputs}

In order to describe the performance of different methods for controlling the spectral radiance of self-luminous displays it is necessary to quantify the photic input to the human circadian system, as well as its associated effects. Several approaches, widely different in scope and predictive power, have been proposed to that end. Their final aim is to relate a physically measurable magnitude, usually the spectral irradiance $E(\lambda)$ at the eye cornea, to the response elicited in the circadian system (e.g. the percentage of melatonin suppression produced under defined experimental conditions). Following the tradition of visual photometry most of the proposed models for quantifying the circadian input $J$ are linear, giving it as a weighted integral of the spectral irradiance:

$$
J=K \int w(\lambda) E(\lambda) \mathrm{d} \lambda
$$

where the weighting function $w(\lambda)$ and the scaling constant $K$ are chosen according to some predefined criterium. An early example of this kind of models is the one proposed by Gall, ${ }^{15,16}$ whose weighting function $w(\lambda)=c(\lambda)$ tries to fit in an approximate way the Brainard et $a l^{17,18}$ and Thapan et $a l^{19}$ action spectra for acute pineal melatonin suppression in healthy subjects under quasi-monochromatic illumination. Based on Gall's $c(\lambda)$ weighting curve several magnitudes like the circadian illuminance $(C I L)$, the circadian efficacy of radiation (CER) and the circadian action factor (CAF) can be defined, for the photopic, mesopic and scotopic ranges. ${ }^{12-15}$

However, a single weighting function seems clearly insufficient for capturing all features of the light-based input to the human circadian system. Besides the intrinsically photosensitive retinal ganglion cells (ipRGC) ${ }^{20}$, the rods and the $S, L$ and $M$ cones play a relevant role in the circadian photic regulation, ${ }^{21}$ through a network of complex interactions whose quantitative details are beginning to be understood but have not been yet fully characterized. In order to allow successful comparison of results it has therefore been suggested that the spectral power distributions used in any experiment should be recorded and that, at least, the individual inputs to these 
individual photoreceptors should be reported. ${ }^{10}$ These inputs can be quantified by the spectral irradiance at the eye cornea weighted by the appropriate $\alpha$-opic functions $N_{\alpha}(\lambda)$ defined in Reference 10, normalized to unit area, and multiplied by a suitable constant in order to be expressed in cyanopic, chloropic, erythropic, melanopic and rhodopic lux. A recent CIE Technical Note ${ }^{22}$ advocates normalizing the $N_{\alpha}(\lambda)$ functions to 1 at their maximum, and expressing the photoreceptor inputs as weighted corneal irradiances, in units of $\mathrm{W} / \mathrm{m}^{2}$, according to the standard practice of the International System of Units (SI).

Determining these inputs is useful for assessing the relative excitation of the photoreceptors involved in circadian regulation, but does not provide by itself any information about the responses that may be elicited. A comprehensive model for predicting these responses was proposed by Rea et $a l^{23}$ in 2005 and refined in subsequent years. ${ }^{24-26}$ With a sound basis on the known neuroanatomy and physiology of the visual and circadian systems, the Rea et al approach makes it possible to calculate the circadian system output (in terms of acute melatonin suppression) as a function of the photic stimulus (the spectral power distribution at the subject's cornea). In addition to the linear contribution from the ipRGC, this model takes into account several kind of nonlinear interactions, by including a cone-based spectral opponency term with an associated rod shunting factor. The model equations make it possible to compute the effective input to the circadian system in terms of circadian light $\left(C L_{A}\right){ }^{24,25}$ a magnitude from which the expected level of acute melatonin suppression (CS) under particular experimental conditions can be calculated with the help of a sigmoid function. ${ }^{26}$ The values of CS usually range from 0 (no melatonin suppression) to a maximum of 0.7 (70\% of melatonin suppression). Here we will report them as percentages, denoted by $C S(\%)$, so that $C S(\%)=100 \times C S$. The model equations correspond to standard observing conditions ${ }^{25}$ characterized by one-hour continuous exposure of the subjects to uniform light fields at the central hours of the night, with undilated pupil sizes of $2.5 \mathrm{~mm}$ diameter.

In this work we used the values of $C L_{A}$ and $C S$ to assess the efficacy of each combination of filters and displays. We also report, as complementary information, the values of the corresponding Gall's parameters and the multi-receptor $\alpha$-opic inputs. These complementary data may provide some additional insights to evaluate the different approaches, since they describe, among others, the relative excitation of the photoreceptors under different spectral power distributions. However, it must be borne in mind that within this framework the Rea et al model's CS is the only magnitude that gives us quantitative information about the response of the circadian system to the exposure to a light source. 


\section{Examples}

With the aim of providing some practical insights about the possibilities of controlling the circadian input produced by self-luminous devices we analyzed a few hardware and software configurations. The results reported here are intended to be illustrative, and should not be taken as an overall evaluation of any product, device, code or application, nor do they imply any kind of endorsement, recommendation or assessment of suitability for any definite purpose. Models and trademarks are mentioned for the sake of information and for allowing any interested reader to reproduce and critically assess these results.

\subsection{Displays and filters}

Two self-luminous displays were used for these measurements, corresponding to a portable computer MacBook Pro (Retina, 15-inch, Mid 2014), equipped with a 332 $\mathrm{mm} \times 208 \mathrm{~mm}$, 15.4-inch diagonal LED-backlit display with $2880 \times 1800$ in-plane switching (IPS) LCD pixels; and a Samsung GT-I8190 cell phone, featuring an active matrix OLED display of $480 \times 800$ pixels.

To the best of our knowledge no specific hardware filters for reducing the circadian input by fitting them to such self-luminous devices were widely available in the market at the time of writing these lines. There is, however, an extensive offer of filters that claim to reduce the amount of blue light reaching the eyes, with the declared main goal of avoiding potential photobiological damage to the retina. Although these claims remain controversial for want of sufficient proof, we included in this study several filters of this kind with the only purpose of assessing up to what point could they be useful for reducing the short-wavelength light exposure at spectral bands known to elicit strong circadian responses. The models chosen were the Reticare M (Medium), H (High) and I (Intensive) filters for smartphones and tablets. ${ }^{27}$

Among the free software applications exisiting for different kind of devices and operating systems at the time of performing these measurements we selected two: f.lux (v 3.10), available for Windows, Mac, Linux, and iPhone/iPad devices ${ }^{28}$ and Softworx's BlueLight Color Filter (v 2.8.2), for Android. ${ }^{29}$

\subsection{Measurement procedure}

The spectral irradiance measurements were made at the Light Pollution Laboratory of the Universidade de Santiago de Compostela using a radiometrically calibrated BlueWave spectrometer (STE-BW-VIS-25, StellarNet, Inc, FL), operating in the 350-1150 $\mathrm{nm}$ range and fitted with cosine correction.

\subsubsection{Portable computer}

The MacBook Pro computer was configured to display a white full screen with the maximum available brightness. The irradiance was measured with the detector 
surface parallel to the display, centred in the middle of the screen, and at an axial distance of $40 \mathrm{~cm}$ from it, corresponding to 2.5 dioptres of accommodation for an emmetropic observer. The detector integration time was set to $2000 \mathrm{~ms}$.

(a) Software filters (f.lux): We measured the spectral irradiance at the detector plane with the f.lux set to correlated colour temperatures (CCT) of 1200, 1900, 2700, 3400, 4200, 5500, 6000 and 6500K, and with no filter (f.lux disabled). Additional measurements at these CCT settings were also made at 30 and $50 \mathrm{~cm}$ from the screen, and in all cases a complementary reading with $750 \mathrm{~ms}$ integration time was taken to assess the consistency of the measured spectra.

(b) Hardware filters (Reticare): The measurements were made with the I, $\mathrm{H}$ and $\mathrm{M}$ Reticare filters, and with no filter. We used filters of size $158 \mathrm{~mm} \times 195 \mathrm{~mm}$, centred on the display, that did not cover its full extent. The remaining area was blocked with a black screen, and the readings were corrected for the different size and viewing angle by scaling the 'No filter' irradiance obtained with this setup to that obtained with 'No filter' in the f.lux case (see above). We consistently obtained a scaling factor of 1.7008 . This factor is smaller than the one that would be obtained from the proportion between the respective solid angles (2.0622) if the source were Lambertian, revealing that the computer display deviates in some degree from this simple behaviour.

\subsubsection{Smartphone}

The irradiance was measured at $25 \mathrm{~cm}$ from the cell phone display, a conventional near-point distance for an emmetropic subject (at 4.0 dioptres of accommodation), with the detector surface centred and parallel to it. The device displayed a full screen white field and its brightness was set at the maximum allowed value. The detector integration time was set to $3000 \mathrm{~ms}$, to ensure a good signal-tonoise ratio even for the maximum attenuation values recorded in this measurement run.

(a) Software filters (Bluelight app): The display was set at the maximum brightness provided by this app and the red colour was chosen for the filter. With these parameters the strength of the filtering effect is determined by the 'opacity' control, which ranges from $0 \%$ (no filtering) to $90 \%$ (maximum filtering). The irradiance was recorded with the opacity parameter set to $0 \%, 15 \%, 30 \%, 45 \%, 60 \%, 75 \%$ and $90 \%$. The baseline spectrum was measured with the app deactivated.

(b) Hardware filters (Reticare): The measurements were made using the I, $\mathrm{H}$, and $\mathrm{M}$ filters, and with no filter, in all cases with the BlueLight app deactivated and at the same distance and integration times as quoted above.

\subsection{Circadian parameters}

From the measured spectral irradiances in $\mathrm{W} \cdot \mathrm{m}^{-2} \cdot \mathrm{nm}^{-1}$ we computed the following parameters for the different combinations of displays and filters: 
- The circadian light input $C L_{A}$, calculated using the Rea et al model ${ }^{25}$ with the bestfitting coefficients corresponding to a lens-corrected melanopsin function peaking at $484 \mathrm{~nm}$.

- The expected control-adjusted melatonin suppression ratio for a standard experimental condition, using the formula for CS from reference 26.

- The cyanopic, chloropic, erythropic, melanopic and rhodopic illuminances, according to the different normalizations proposed in references 10 and 22 .

- The photopic illuminance (PIL), photopic efficacy of radiation (LER), circadian efficacy of radiation (CER), circadian action factor (CAF) and circadian illuminance $(C I L) .{ }^{12,13,15}$

- The overall irradiance, and the chromaticity $(x, y)$ coordinates in the CIE 1931 colour space.

\subsection{Results}

The spectral irradiance measured at $40 \mathrm{~cm}$ from the computer display is shown in Figures 1 (with the f.lux software filter) and 2 (with the Reticare hardware filters). In both cases the dots correspond to the unfiltered display. The corresponding circadian magnitudes are listed in Tables 1 and 2, respectively. The results with the smartphone display at $25 \mathrm{~cm}$ distance are shown in an analogous way in Figures 3 (with the Bluelight software filter) and 4 (with the Reticare ones), and the associated circadian magnitudes are listed in Tables 3 and 4. Figure 5 shows the values of $C L_{A}$ for these combinations of displays and filters.

The positions in the CIE 1931 chromaticity space of the light arising in each configuration are shown in Figure 6. They correspond to the computer screen (filled symbols) and the smartphone display (open symbols), using software filters (squares) and hardware filters (circles). Triangles correspond to unfiltered light.

Dimming the screens without changing the shape of their spectral power distributions is equivalent to scaling down the measured irradiances by the brightness reduction factor. Two reduced brightness levels were considered, corresponding to $50 \%$ and $25 \%$ of the maximum radiance for each device. Only the results for $C L_{A}$ and $C S$ are reported in Table 5. Note that the remaining magnitudes included in Tables 1 to 4 either depend linearly on the absolute value of the irradiance or do not depend at all on it. For those that depend linearly, in the case of dimming the screens their values will just be scaled to 0.5 or 0.25 of the tabulated ones: this is the case of the $\alpha$-opic illuminances or weighted irradiances, the photopic illuminance $(P I L)$, the circadian illuminance $(C I L)$ and the total irradiance. Magnitudes that are ratios of weighted irradiances such as the $L E R, C E R, C A F$ and the chromaticity coordinates remain invariant. Since in general $C L_{A}$ and $C S$ are nonlinear functions of the irradiance their values when dimming the screens will not necessarily be proportional to the dimming factor; however, here they are very closely proportional, because for the irradiance 
levels recorded in our measurements the Rea et al equations for $C L_{A}$ (once the sign of the spectral opponency term is settled) behave in an approximately linear way.

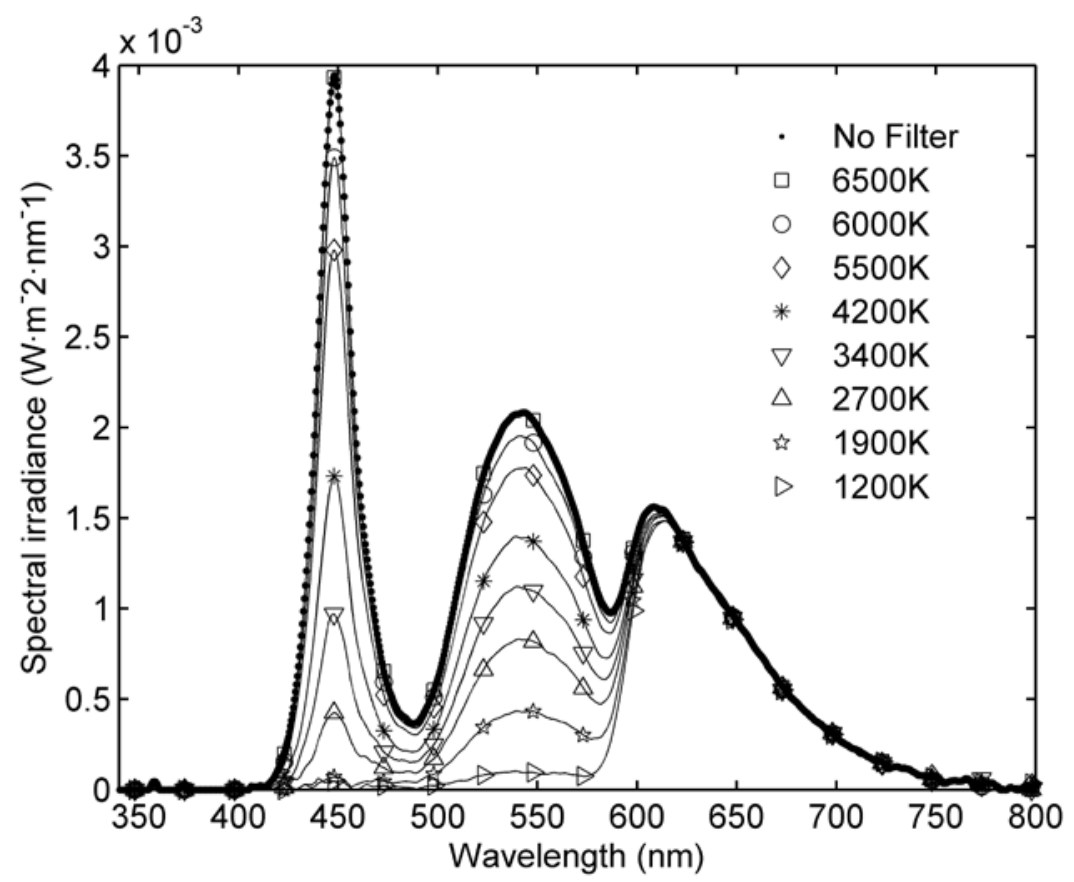

Figure 1. Computer display with f.lux software filters: Spectral irradiance $\left(\mathrm{W} \cdot \mathrm{m}^{-2} \cdot \mathrm{nm}^{-1}\right)$ measured at $40 \mathrm{~cm}$ from the device. The dotted line corresponds to the unfiltered display, and the remaining curves correspond to the use of a software filter with different CCT settings.

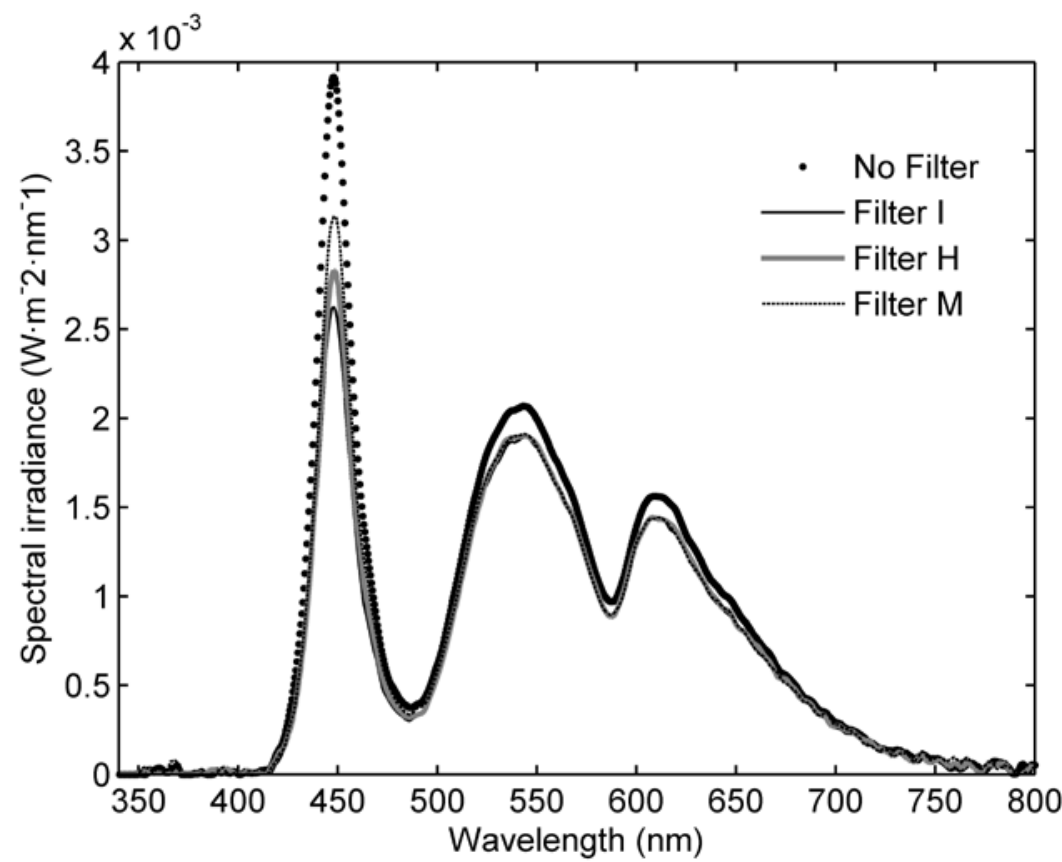

Figure 2. Computer display with Reticare hardware filters: Spectral irradiance $\left(\mathrm{W} \cdot \mathrm{m}^{-2} \cdot \mathrm{nm}^{-1}\right)$ measured at $40 \mathrm{~cm}$ from the device. The dotted line corresponds to the 
unfiltered display, and the remaining curves correspond to the use of a hardware filter with different filtering effects (M: Medium, H: High, I: Intensive).

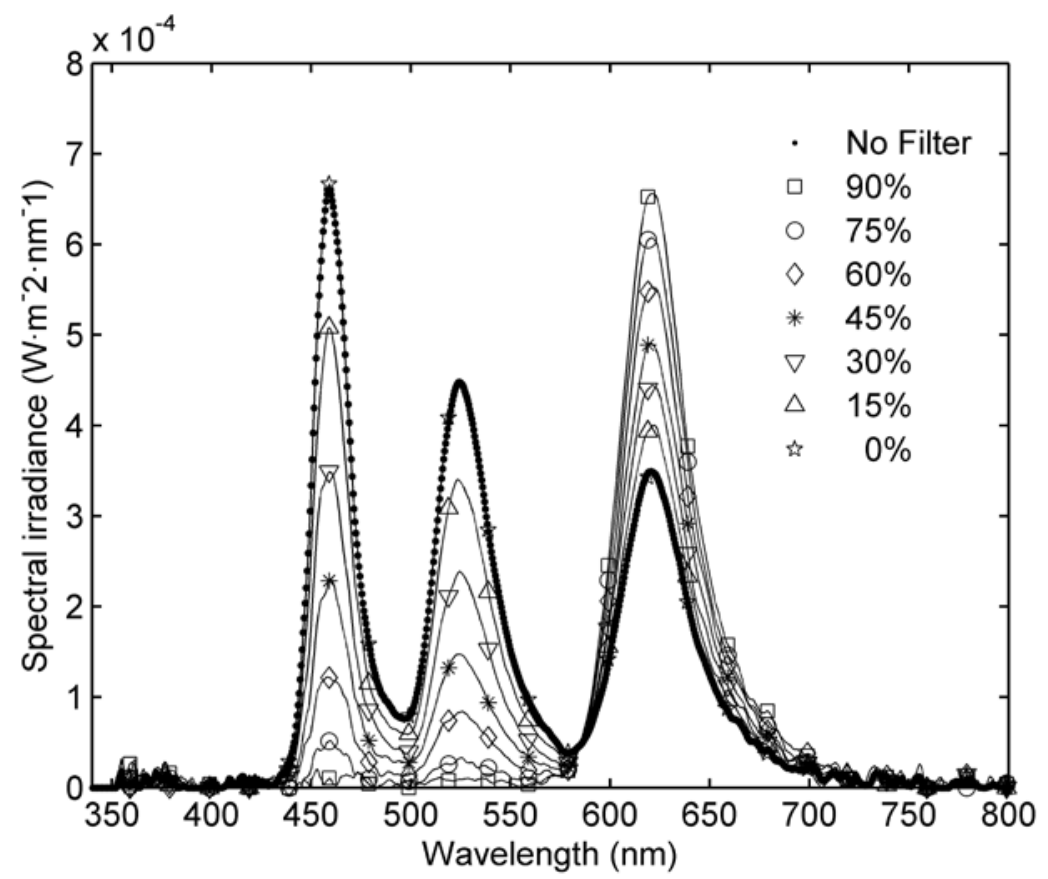

Figure 3. Smartphone display with BlueLight software filters: Spectral irradiance $\left(\mathrm{W} \cdot \mathrm{m}^{-2} \cdot \mathrm{nm}^{-1}\right)$ measured at $25 \mathrm{~cm}$ from the device. The dotted line corresponds to the unfiltered display, and the remaining curves correspond to the use of a software filter with different 'opacity' settings.

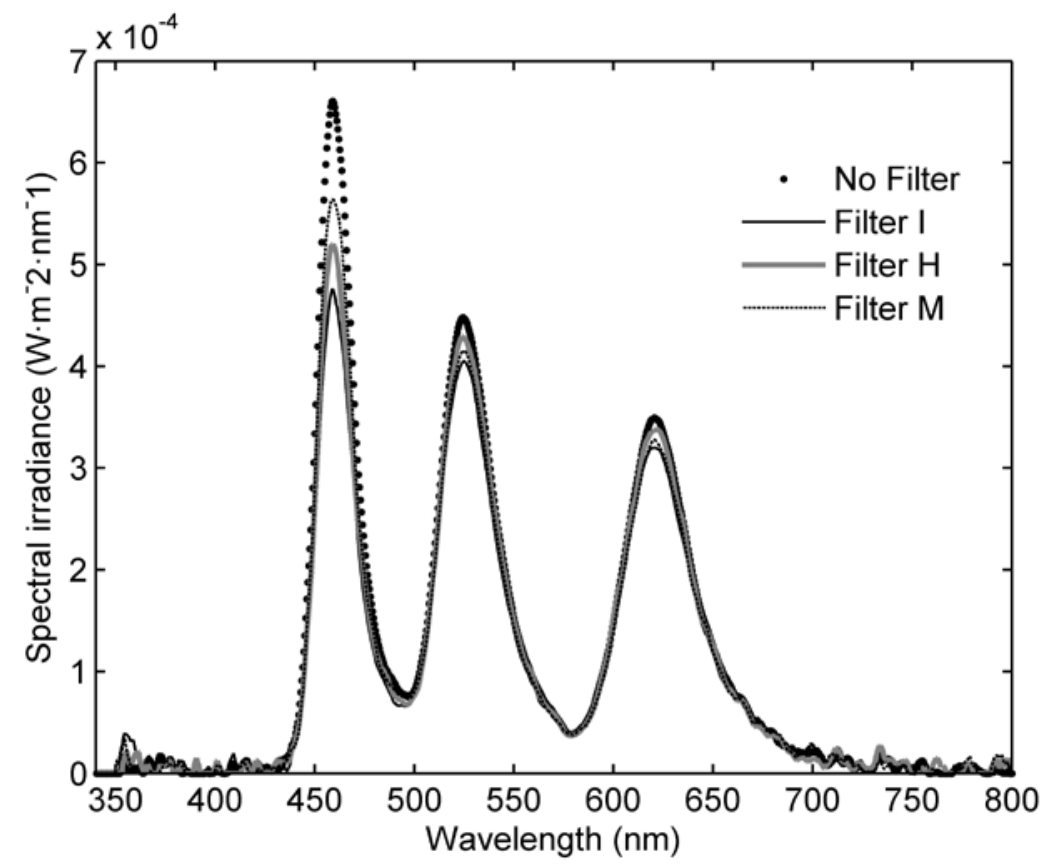

Figure 4. Smartphone display with Reticare hardware filters: Spectral irradiance $\left(\mathrm{W} \cdot \mathrm{m}^{-2} \cdot \mathrm{nm}^{-1}\right)$ measured at $25 \mathrm{~cm}$ from the device. The dotted line corresponds to the 
unfiltered display, and the remaining curves correspond to the use of a hardware filter with different filtering effects (M: Medium, H: High, I: Intensive).
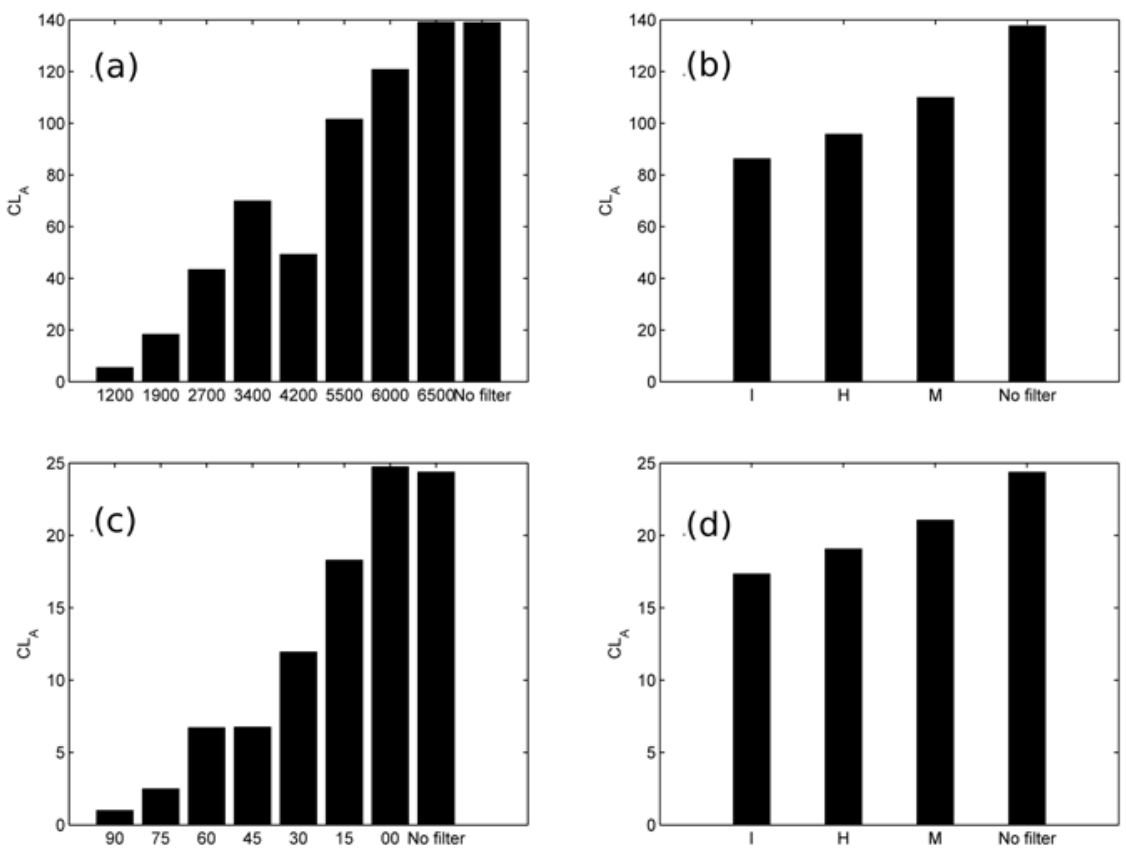

Figure 5. $C L_{A}$ values for (a) computer display with f.lux filters. The $\mathrm{X}$ axis labels correspond to the CCT parameter in Kelvin; (b) computer display with Reticare filters (I:intense, H:high, M:medium); (c) smartphone display with BlueLight filters. The $\mathrm{X}$ axis labels correspond to the opacity (equal to 1-Transmittance) expressed in percentage; (d) smartphone display with Reticare filters.

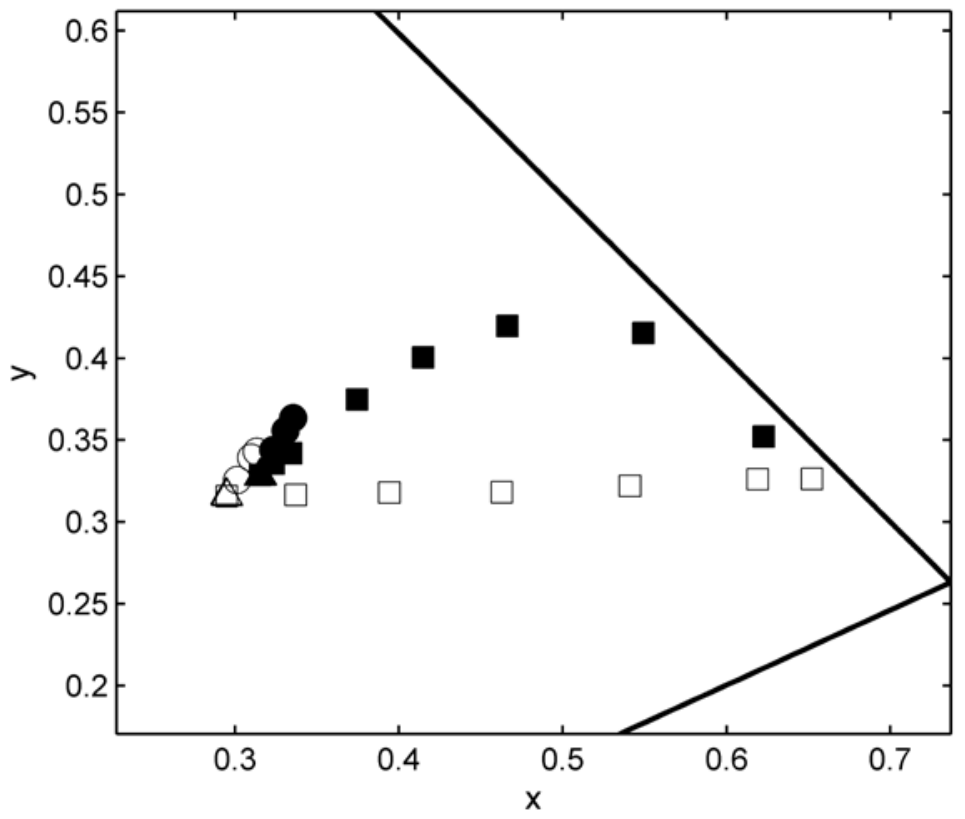


Figure 6. Chromaticity coordinates in the CIE 1931 chromaticity space of the light emitted by the computer screen (filled symbols) and the smartphone display (open symbols), using software filters (squares) and hardware filters (circles). Triangles correspond to unfiltered light. Lower CCT or high opacity values in software filters displace the chromaticity point towards the monochromatic border of the diagram, in our case towards the red region.

\section{Discussion}

The results obtained here show that software filters are able to reduce significantly the circadian light input $\left(C L_{A}\right)$ and the expected melatonin suppression $(C S)$ in comparison with unfiltered light, specially if low CCT settings (f.lux) or high opacity values (Bluelight) are used. The same can be said about other parameters directly related to the ipRGC sensitivity band, such as the melanopic irradiance or the Circadian Action Factor (CAF). As a trade off, when the settings used in these software filters approach their strongest values (lowest CCTs or highest opacities, respectively), the chromaticity coordinates of the light emitted by the displays are displaced towards the monochromatic border of the CIE diagram, in our case towards the red region of the spectrum. The hardware filters analyzed in this study, in turn, keep the chromaticity point in the high CCT white region of the chromaticity space, but at the expense of a relatively weak filtering effect, as can be seen in the rather modest reduction achieved in the $C L_{A}, C S$ or $C A F$ values.

The use of colour filters of any kind unavoidably changes the screen appearance, and trading-off circadian harmlessness against visual performance may prove to be a demanding issue. Moderate colour changes may soon go unnoticed when performing visual tasks that are not critically colour-dependent, e.g. reading text documents, writting e-mails, searching the web, messaging or interacting through different social networking platforms. Conversely, video watching requires, in most cases, disabling the filters or considerably softening their settings. Different population segments may have different patterns of use and additional studies are required to establish the optimum configurations of self-luminous devices, especially for those age groups that may spend a relatively bigger fraction of time on film and video watching activities. An easy procedure for toggling between night and video mode settings would surely ease acceptance.

Neutral filtering (dimming the screens) is a practical method to help reducing the circadian load without significantly affecting colour rendition. As shown in Tables 1 and 5 , dimming the brightness of the computer screen to $50 \%$ of its maximum gives rise to a melatonin suppression of $9.89 \%$, roughly equivalent to using a software filter in the $3400 \mathrm{~K} \mathrm{CCT}$ range. Dimming it to the $25 \%$ allows to reduce the suppression to 
$4.97 \%$, equivalent to using a software filter with CCT below $2700 \mathrm{~K}$. Note that the photopic illuminance provided by the f.lux filter, in the configuration used in our measurements, also decreases with increasing CCT: The filter works by attenuating the medium and short wavelength regions of the spectrum while keeping almost constant the power emitted at long wavelengths (Figure 1). As the filtering effect becomes more intense the overall illuminance is progressively reduced. That reduction, however, is proportionally smaller than the one required if only dimming the screen to achieve comparable CS values (see the values of the photopic illuminance (PIL) in Table 1). Regarding the smartphone display (Tables 3 and 5), dimming it to 50\% (CS(\%)=1.66) amounts to using a $30 \%$ opacity factor, and setting it to $25 \%(C S(\%)=0.78)$ is equivalent to using an opacity factor larger than $60 \%$. Interestingly, the BlueLight software filter used in these measurements partially compensates for the loss of brightness associated with the attenuation of the short wavelengths by increasing the power emitted in the long-wavelength regions of the spectrum, as can be seen in the spectral power distributions shown in Figure 3.

As stated above, the experimental results presented in this work are only meant to illustrate the performance of some available software and hardware filters when it comes to modifying the spectral content of the light emitted by self-luminous displays. As such, this is not an overall assessment of the performance of these particular products and, of course, no claim is made for completeness. Besides the reduced sample of displays and filtering methods, this study has other relevant limitations.

We restricted ourselves to analyze the light that reaches the observer from the devices, without including the effects of other ambient light sources that may be present in his or her environment. In case of using the devices within illuminated rooms this additional irradiance has to be taken into account, as reported in reference 12. The presence of ambient light aggravates the effects produced by the screens and must be included in any assessment of the overall nocturnal lightscape. As a matter of fact, the circadian effects elicited by the ambient lights may be comparable or even more relevant than the ones produced by the displays. It is worth noting that when several light sources are simultaneoulsy present their circadian effects cannot as a general rule be analyzed separately: Due to the non-linear dependence of $C L_{A}$ and $C S$ on the irradiance, the correct procedure for evaluating the combined effect of ambient lights and displays is first adding their spectral irradiances at the subject's cornea, and subsequently applying the Rea et al equations to the resulting irradiance in order to obtain the increased values of $C L_{A}$ and $C S$.

For the sake of precision let us add that albeit in general neither $C L_{A}$ nor $C S$ are linear functions of the spectral irradiance, there are some exceptions. $C L_{A}$ becomes approximately linear in the limits of very low or very high irradiances if the sign of the spectral opponency term ${ }^{25}$ is positive, and is always linear when the sign of that term is 
negative: in that case, then $C L_{A}$ is given just by the linear contribution arising from the ipRGC. ${ }^{25}$ CS, in turn, behaves in an approximately linear way in the vicinity of the inflection point of the sigmoid curve.

Another limitation of this work is the implicit assumption of a constant exposure of the observer to an uniformly illuminated field. This is partly motivated by the fact that the Rea et al model equations used in this paper are valid for a given exposure time under constant irradiance at the cornea. The other reported parameters are time-independent and only provide information about the photoreceptor inputs, but not about the time course of the responses. In everyday indoor or outdoor settings people are obviously subjected to a continuously changing lightscape, strongly dependent on the direction of gaze, and the same applies for people using selfluminous screens for watching movies or surfing the web during variable periods of time. We feel, however, that the results provided here can be useful as a starting point for assessing the relative efficacy of the different approaches for reducing the circadian load.

It shall also be noted that the reported results correspond to two standardized viewing distances, $40 \mathrm{~cm}$ in case of computer screens and $25 \mathrm{~cm}$ in case of phones. The actual distances people use to handle their devices may of course vary, depending on the task, observer's ammetropy, accommodation amplitude, and any optical correction worn. They may tend to be larger for undercorrected presbyopic adults, and they will often be shorter -especially in case of smartphones- for uncorrected moderate myopes and emmetropic young people. Shorter viewing distances will translate into higher levels of corneal irradiance.

No absolute product comparison between the particular software and hardware filters used in this study should be made from our data, since these filters were not designed by their manufacturers for producing comparable effects. It can be anticipated that appropriately designed hardware filters could achieve a performance similar to that obtained with the software applications at low CCTs or high opacities. Hardware filters also offer the possibilty of performing narrow-band filtering operations, which can be of interest for efficiently blocking some well defined wavelength regions without affecting others. However, software filters have an exclusive appealing feature, shown in Figure 3: Whereas hardware filters are unavoidably passive, so their transmittance is always less than unity for all spectral wavelengths, software filters can be active, enhancing the light emission in some spectral regions while at the same time attenuating it in others. This offers additional degrees of freedom for implementing appropriate colour compositions at various levels of irradiance and for tailoring the light emission to minimize the unwanted circadian effects produced by the use of these devices.

Overall, both approaches can be useful for the intended task of keeping the circadian stimulus to the users within predefined limits. However, the intrinsic 
features of software filters make them in principle advantageous: They do not require the consumption of raw materials, can be easily adjusted for different lighting needs, can be distributed to the end users through habitual download channels and can be continuosly updated to adjust their performance to the best available knowledge of the circadian effects of the different spectral compositions and intensities of light. We consider that such kind of tools should be a standard feature of any off-the-shelf electronic device equiped with self-luminous displays.

As a final remark, it is important to note that the negative effects of light from self-luminous displays dealt with in this paper specifically concern their use at night, particularly shortly before bedtime, concident with the onset of melatonin production and an increased phase shifting sensitivity. But the light produced by self-luminous devices could potentially also have beneficial effects, if used at appropriate times along the circadian cycle: As an anonymous reviewer correctly pointed out, tailored spectral power distributions could probably be instrumental in achieving suitable entrainment stimuli in the mornings. Although phone screens seem to fall somewhat short of having enough radiant power for this application, computer screens are an interesting possibility that deserve further study.

\section{Conclusions}

Both hardware filters fitted onto the displays and software applications that allow modifying the spectral content of the displayed images are efficacious means to reduce the circadian stimulus suffered by the users of self-luminous devices at nighttime. Overall, software applications seem to offer the best trade-offs for a flexible and tailored control of the spectral irradiance of the light emitted by existing devices. We submit that such tools should be included as a standard feature in any off-the-shelf consumer product equiped with self-luminous displays, much in the same way that traditional brightness and contrast controls are, and that their default settings should be established and updated according to the best available knowledge about the circadian effects of the different spectral compositions and intensities of light.

\section{Funding}

This work has been funded in part by the Spanish Ministry of Economy and Competitiveness (grant DPI2013-43220-R) and FEDER, and was developed within the framework of the Spanish Network for Light Pollution Studies. 


\section{Acknowledgements}

We acknowledge the insightful comments and suggestions made by two anonymous reviewers.

\section{References}

1. Chang Y-L, Lu Z-H. White organic light-emitting diodes for solid-state lighting. Journal of Display Technology, 2013; 9: 459 - 468.

2. Zeitzer JM, Dijk DJ, Kronauer R, Brown E, Czeisler C. Sensitivity of the human circadian pacemaker to nocturnal light: Melatonin phase resetting and suppression. Journal of Physiology 2000; 526: 695-702.

3. Gooley JJ, Chamberlain K, Smith KA, Khalsa SBS, Rajaratnam SMW, Reen EV, Zeitzer JM, Czeisler CA, Lockley SW. Exposure to room light before bedtime suppresses melatonin onset and shortens melatonin duration in humans. Journal of Clinical Endocrinology and Metabolism, 2011, 96(3): E463-E472

4. Dijk D-J, Archer SN. Light, sleep, and circadian rhythms: Together again. PLoS Biology 2009:7 e1000145.

5. Czeisler CA. Perspective: Casting light on sleep deficiency. Nature 2013; 497: S13.

6. Dijk DJ. Why do we sleep so late? Journal of Sleep Research 2013; 22: 605-606.

7. Figueiro $M$, Overington D. Self-luminous devices and melatonin suppression in adolescents. Lighting Research and Technology, published online before print 6 May 2015, doi: 10.1177/1477153515584979.

8. American Medical Association. Light pollution: Adverse health effects of nighttime lighting. In Proceedings of the American Medical Association House of Delegates, 161st Annual Meeting, Chicago, IL, 2012, pp 265-279.

9. Stevens RG, Brainard GC, Blask DE, Lockley SW, Motta ME. Adverse health effects of nighttime lighting: Comments on American Medical Association policy statement. American Journal of Preventive Medicine 2013; 45: 343-346.

10. Lucas RJ, Peirson SN, Berson DM, Brown TM, Cooper HM, Czeisler CA, Figueiro MG, Gamlin PD, Lockley SW, O'Hagan HB, Price LLA, Provencio I, Skene DJ, Brainard GC. Measuring and using light in the melanopsin age. Trends in Neurosciences 2014; 37: 1-9.

11. Bonmati-Carrion MA, Arguelles-Prieto $R$, Martinez-Madrid MJ, Reiter $R$, Hardeland R, Rol MA, Madrid JA. Protecting the melatonin rhythm through circadian healthy light exposure. International Journal of Molecular Sciences 2014; 15: 23448-23500. 
12. Oh JH, Yoo H, Park HK, Do YR. Analysis of circadian properties and healthy levels of blue light from smartphones at night. Scientific Reports 2015; 11325. doi: $10.1038 /$ srep11325.

13. Oh JH, Yang SJ, Do YR. Healthy, natural, efficient and tunable lighting: fourpackage white LEDs for optimizing the circadian effect, color quality and vision performance. Light: Science and Applications 2014; 3, e141. doi:10.1038/Isa.2014.22

14. Zukauskas A, Vaicekauskas R, Vitta P. Optimization of solid-state lamps for photobiologically friendly mesopic lighting. Applied Optics 2011; 51: 84238432.

15. Gall D. Die messung circadianer strahlungsgrößen. In: Viertes Symposium Licht und Gesundheit, 26-27 February 2004, pp. 120-138. Berlin: Paul Kistmacher, 2004.

16. Gall D, Bieske K. Definition and measurement of circadian radiometric quantities. In: Light and Health - Non-Visual Effects : Proceedings of the CIE Symposium '04 , 30 September - 2 October 2004, Vienna, Austria, pp. 129-132. Vienna : CIE, 2004.

17. Brainard GC, Hanifin JP, Greeson JM, Byrne B, Glickman G, Gerner E, Rollag MD. Action spectrum for melatonin regulation in humans: evidence for a novel circadian photoreceptor. Journal of Neuroscience 2001; 21: 6405-6412.

18. Brainard GC, Sliney D, Hanifin JP, Glickman G, Byrne B, Greeson JM, Jasser S, Gerner E, Rollag MD. Sensitivity of the human circadian system to shortwavelength (420-nm) light. Journal of Biological Rhythms 2008; 23: 379-386.

19. Thapan K, Arendt J, Skene DJ. An action spectrum for melatonin suppression: evidence for a novel non-rod, non-cone photoreceptor system in humans. Journal of Physiology 2001; 535: 261-267.

20. Berson DM, Dunn FA, Takao M. Phototransduction by retinal ganglion cells that set the circadian clock. Science 2002; 295: 1070-1073.

21. Dacey DM, Liao H-W, Peterson BB, Robinson FR, Smith VC, Pokorny J, Yau K-W, Gamlin PD. Melanopsin-expressing ganglion cells in primate retina signal colour and irradiance and project to the LGN. Nature 2005; 433: 749-754.

22. Commission Internationale de I'Eclairage. CIE Publication TN 003:2015 Report on the First International Workshop on Circadian and Neurophysiological Photometry, 2013. Vienna : CIE, 2015.

23. Rea MS, Figueiro MG, Bullough JD, Bierman A. A model of phototransduction by the human circadian system. Brain Research Reviews 2005; 50: 213-228.

24. Rea M, Figueiro MG, Bierman A, Bullough JD. Circadian light. Journal of Circadian Rhythms 2010: 8, doi:10.1186/1740-3391-8-2. 
25. Rea MS, Figueiro MG, Bierman A, Hamner R. Modeling the spectral sensitivity of the human circadian system. Lighting Research and Technology 2012; 44: 386-396. Corrigendum: Lighting Research and Technology 2012; 44: 516.

26. Rea MS, Figueiro MG. A working threshold for acute nocturnal melatonin suppression from "white" light sources used in architectural applications. Journal of Carcinogenesis and Mutagenesis 2013; 4: 150.

27. Reticare. Protect your eyes. Retrieved 29 August 2015 from www.reticare.com.

28. f.lux. Software to make your life better. Retrieved 15 August 2015 from https://justgetflux.com/.

29. Softworkx. Bluelight Screen Filter app. Retrieved June 2015 from http://m.downloadatoz.com/apps/com.softworx. bluelight,1237165.html 
Table 1. Circadian magnitudes and stimulus characteristics corresponding to the spectral irradiance measured at $40 \mathrm{~cm}$ from the computer display with software filters (Figure 1).

\begin{tabular}{lrrrrrrrrr} 
Filter (CCT) & $\mathbf{1 2 0 0}$ & $\mathbf{1 9 0 0}$ & $\mathbf{2 7 0 0}$ & $\mathbf{3 4 0 0}$ & $\mathbf{4 2 0 0}$ & $\mathbf{5 5 0 0}$ & $\mathbf{6 0 0 0}$ & $\mathbf{6 5 0 0}$ & No Filter \\
\hline$C_{A}$ & 5.45 & 18.33 & 43.45 & 69.97 & 49.27 & 101.60 & 120.89 & 139.20 & 138.98 \\
CS (\%) & 0.69 & 2.56 & 6.27 & 9.99 & 7.11 & 14.05 & 16.33 & 18.36 & 18.33 \\
\hline Cya-lux & 1.62 & 2.45 & 13.41 & 29.02 & 50.84 & 88.22 & 102.38 & 115.56 & 115.46 \\
Mel-lux & 2.87 & 10.21 & 23.16 & 36.24 & 51.48 & 75.68 & 85.52 & 93.84 & 93.71 \\
Rod-lux & 4.49 & 15.18 & 30.78 & 44.76 & 59.99 & 83.06 & 92.86 & 100.81 & 100.70 \\
Chl-lux & 13.55 & 26.53 & 43.19 & 56.59 & 70.18 & 89.64 & 98.33 & 105.03 & 104.94 \\
Ery-lux & 28.18 & 39.59 & 53.88 & 64.91 & 75.91 & 91.30 & 98.31 & 103.60 & 103.54 \\
\hline Cya $\left(\mathrm{mW} / \mathrm{m}^{2}\right)$ & 1.2 & 1.8 & 10.1 & 21.9 & 38.4 & 66.6 & 77.2 & 87.2 & 87.1 \\
Mel $\left(\mathrm{mW} / \mathrm{m}^{2}\right)$ & 3.5 & 12.3 & 27.8 & 43.5 & 61.8 & 90.9 & 102.7 & 112.7 & 112.6 \\
Rod $\left(\mathrm{mW} / \mathrm{m}^{2}\right)$ & 6.4 & 21.5 & 43.6 & 63.4 & 85.0 & 117.8 & 131.6 & 142.9 & 142.8 \\
Chl $\left(\mathrm{mW} / \mathrm{m}^{2}\right)$ & 21.8 & 42.8 & 69.7 & 91.3 & 113.2 & 144.6 & 158.6 & 169.4 & 169.2 \\
Ery $\left(\mathrm{mW} / \mathrm{m}^{2}\right)$ & 48.7 & 68.4 & 93.1 & 112.1 & 131.1 & 157.7 & 169.8 & 179.0 & 178.9 \\
\hline Irrad $\left(\mathrm{mW} / \mathrm{m}^{2}\right)$ & 117.5 & 141.0 & 179.6 & 214.0 & 254.0 & 315.5 & 340.8 & 361.9 & 362.1 \\
LER & 209.69 & 270.82 & 304.02 & 312.89 & 310.90 & 302.60 & 302.59 & 300.63 & 300.29 \\
CER & 15.29 & 36.75 & 83.59 & 125.33 & 165.42 & 213.33 & 226.34 & 237.47 & 237.03 \\
CAF & 0.07 & 0.14 & 0.27 & 0.40 & 0.53 & 0.70 & 0.75 & 0.79 & 0.79 \\
PIL (Ix) & 24.64 & 38.17 & 54.60 & 66.97 & 78.98 & 95.47 & 103.13 & 108.80 & 108.74 \\
CIL & 1.80 & 5.18 & 15.01 & 26.82 & 42.02 & 67.30 & 77.14 & 85.95 & 85.84 \\
\hline$x$ & 0.6225 & 0.5491 & 0.4663 & 0.4148 & 0.3745 & 0.3342 & 0.3237 & 0.3154 & 0.3155 \\
$y$ & 0.3521 & 0.4154 & 0.4197 & 0.4006 & 0.3747 & 0.3420 & 0.3356 & 0.3287 & 0.3287 \\
\hline
\end{tabular}


Table 2. Circadian magnitudes and stimulus characteristics corresponding to the spectral irradiance measured at $40 \mathrm{~cm}$ from the computer display fitted with hardware filters (Figure 2).

\begin{tabular}{lrrrr} 
Filter model & \multicolumn{1}{c}{ I } & \multicolumn{1}{c}{ H } & \multicolumn{1}{c}{ M } & No Filter \\
\hline$C L_{A}$ & 86.26 & 95.77 & 109.97 & 139.04 \\
CS (\%) & 12.13 & 13.33 & 15.06 & 18.34 \\
\hline Cya-lux & 78.63 & 84.84 & 93.40 & 115.49 \\
Mel-lux & 74.07 & 77.42 & 81.96 & 93.42 \\
Rod-lux & 83.83 & 86.51 & 89.69 & 100.30 \\
Chl-lux & 92.07 & 93.81 & 95.18 & 104.54 \\
Ery-lux & 92.57 & 93.88 & 94.44 & 103.30 \\
\hline Cya $\left(\mathrm{mW} / \mathrm{m}^{2}\right)$ & 59.3 & 64.0 & 70.5 & 87.1 \\
Mel $\left(\mathrm{mW} / \mathrm{m}^{2}\right)$ & 89.0 & 93.0 & 98.5 & 112.2 \\
Rod $\left(\mathrm{mW} / \mathrm{m}^{2}\right)$ & 118.8 & 122.6 & 127.1 & 142.2 \\
Chl $\left(\mathrm{mW} / \mathrm{m}^{2}\right)$ & 148.5 & 151.3 & 153.5 & 168.6 \\
Ery $\left(\mathrm{mW} / \mathrm{m}^{2}\right)$ & 159.9 & 162.2 & 163.2 & 178.5 \\
\hline Irrad $\left(\mathrm{mW} / \mathrm{m}^{2}\right)$ & 307.3 & 316.0 & 324.1 & 363.2 \\
LER & 319.33 & 314.29 & 307.17 & 298.42 \\
CER & 204.22 & 211.10 & 222.73 & 236.02 \\
CAF & 0.64 & 0.67 & 0.72 & 0.79 \\
PIL $(\mathrm{Ix})$ & 98.13 & 99.32 & 99.56 & 108.40 \\
CIL & 62.76 & 66.71 & 72.19 & 85.73 \\
\hline$x$ & 0.3355 & 0.3308 & 0.3233 & 0.3159 \\
$y$ & 0.3636 & 0.3559 & 0.3441 & 0.3280 \\
\hline & & & &
\end{tabular}


Table 3. Circadian magnitudes and stimulus charateristics corresponding to the spectral irradiance measured at $25 \mathrm{~cm}$ from the smartphone display using software filters (Figure 3).

\begin{tabular}{lrrrrrrrr} 
Filter (opacity) & $\mathbf{9 0 \%}$ & $\mathbf{7 5 \%}$ & $\mathbf{6 0 \%}$ & $\mathbf{4 5 \%}$ & $\mathbf{3 0 \%}$ & $\mathbf{1 5 \%}$ & $\mathbf{0 \%}$ & No Filter \\
\hline CL A $_{\text {CS }(\%)}$ & 0.99 & 2.50 & 6.72 & 6.76 & 11.94 & 18.30 & 24.75 & 24.38 \\
\hline Cya-lux & 0.11 & 0.29 & 0.87 & 0.87 & 1.62 & 2.56 & 3.52 & 3.46 \\
Mel-lux & 0.45 & 1.07 & 2.90 & 5.16 & 7.98 & 11.44 & 14.95 & 14.68 \\
Rod-lux & 0.49 & 1.25 & 3.38 & 6.04 & 9.46 & 13.60 & 17.80 & 17.59 \\
Chl-lux & 0.73 & 1.46 & 3.47 & 5.97 & 9.27 & 13.24 & 17.27 & 17.07 \\
Ery-lux & 3.46 & 3.81 & 5.10 & 6.72 & 9.08 & 11.92 & 14.80 & 14.62 \\
\hline Cya $\left(\mathrm{mW} / \mathrm{m}^{2}\right)$ & 8.71 & 8.49 & 8.89 & 9.41 & 10.65 & 12.20 & 13.77 & 13.59 \\
Mel $\left(\mathrm{mW} / \mathrm{m}^{2}\right)$ & 0.3 & 0.8 & 2.2 & 3.9 & 6.0 & 8.6 & 11.3 & 11.1 \\
Rod $\left(\mathrm{mW} / \mathrm{m}^{2}\right)$ & 0.6 & 1.5 & 4.1 & 7.3 & 11.4 & 16.3 & 21.4 & 21.1 \\
Chl $\left(\mathrm{mW} / \mathrm{m}^{2}\right)$ & 1.0 & 2.1 & 4.9 & 8.5 & 13.1 & 18.8 & 24.5 & 24.2 \\
Ery $\left(\mathrm{mW} / \mathrm{m}^{2}\right)$ & 5.6 & 6.1 & 8.2 & 10.8 & 14.6 & 19.2 & 23.9 & 23.6 \\
\hline Irrad $\left(\mathrm{mW} / \mathrm{m}^{2}\right)$ & 15.0 & 14.7 & 15.4 & 16.3 & 18.4 & 21.1 & 23.8 & 23.5 \\
LER & 32.9 & 31.8 & 33.6 & 35.5 & 39.8 & 45.4 & 51.1 & 50.5 \\
CER & 220.55 & 226.61 & 233.54 & 243.29 & 255.86 & 266.23 & 274.26 & 274.17 \\
CAF & 11.66 & 30.84 & 80.70 & 136.53 & 189.72 & 239.20 & 278.18 & 277.85 \\
PIL $(\mathrm{Ix})$ & 0.05 & 0.14 & 0.35 & 0.56 & 0.74 & 0.90 & 1.01 & 1.01 \\
CIL & 7.26 & 7.21 & 7.84 & 8.64 & 10.20 & 12.09 & 14.019 & 13.84 \\
\hline$x$ & 0.38 & 0.98 & 2.71 & 4.85 & 7.56 & 10.86 & 14.22 & 14.02 \\
\hline$y$ & 0.6521 & 0.6189 & 0.5410 & 0.4627 & 0.3942 & 0.3370 & 0.295 & 0.2948 \\
\hline & 0.3263 & 0.3261 & 0.3221 & 0.3181 & 0.3179 & 0.3166 & 0.3157 & 0.3164 \\
\hline
\end{tabular}


Table 4. Circadian magnitudes and stimulus characteristics corresponding to the spectral irradiance $\left(\mathrm{W} \cdot \mathrm{m}^{-2} \cdot \mathrm{nm}^{-1}\right)$ produced with hardware filters by the smartphone display at $25 \mathrm{~cm}$ from the observer's eye (Figure 4).

\begin{tabular}{lrrrr} 
Filter & I & H & M & No Filter \\
\hline$C L_{A}$ & 17.35 & 19.07 & 21.05 & 24.38 \\
CS $(\%)$ & 2.42 & 2.67 & 2.97 & 3.46 \\
\hline Cya-lux & 10.87 & 11.88 & 12.81 & 14.68 \\
Mel-lux & 14.37 & 15.47 & 15.90 & 17.59 \\
Rod-lux & 14.44 & 15.48 & 15.61 & 17.07 \\
Chl-lux & 12.91 & 13.76 & 13.58 & 14.62 \\
Ery-lux & 12.33 & 13.06 & 12.74 & 13.59 \\
\hline Cya $\left(\mathrm{mW} / \mathrm{m}^{2}\right)$ & 8.2 & 9.0 & 9.7 & 11.1 \\
Mel $\left(\mathrm{mW} / \mathrm{m}^{2}\right)$ & 17.3 & 18.6 & 19.1 & 21.1 \\
Rod $\left(\mathrm{mW} / \mathrm{m}^{2}\right)$ & 20.5 & 21.9 & 22.1 & 24.2 \\
Chl $\left(\mathrm{mW} / \mathrm{m}^{2}\right)$ & 20.8 & 22.2 & 21.9 & 23.6 \\
Ery $\left(\mathrm{mW} / \mathrm{m}^{2}\right)$ & 21.3 & 22.6 & 22.0 & 23.5 \\
\hline Irrad $\left(\mathrm{mW} / \mathrm{m}^{2}\right)$ & 44.2 & 46.9 & 46.8 & 50.5 \\
LER & 286.64 & 286.41 & 278.35 & 274.17 \\
CER & 248.04 & 253.50 & 266.77 & 277.85 \\
CAF & 0.86 & 0.88 & 0.96 & 1.01 \\
PIL $(\mathrm{Ix})$ & 12.67 & 13.42 & 13.02 & 13.84 \\
CIL & 10.96 & 11.88 & 12.48 & 14.02 \\
\hline$x$ & 0.3133 & 0.3099 & 0.3013 & 0.2948 \\
$y$ & 0.3429 & 0.3394 & 0.3257 & 0.3164 \\
\hline
\end{tabular}

Table 5. $C L_{A}$ and $C S$ values corresponding to dimming the unfiltered displays, setting them to levels $50 \%$ and $25 \%$ of the maximum allowed brightness for each device.

\begin{tabular}{lrrr} 
Device/Brightness & $\mathbf{1 0 0 \%}$ & $\mathbf{5 0 \%}$ & $\mathbf{2 5 \%}$ \\
\hline Computer & & & \\
$C L_{A}$ & 138.98 & 69.21 & 34.54 \\
$C S(\%)$ & 18.33 & 9.89 & 4.97 \\
\hline Smartphone & & & \\
$C L_{A}$ & 24.38 & 12.18 & 6.09 \\
$C S(\%)$ & 3.46 & 1.66 & 0.78 \\
\hline
\end{tabular}

